\section{Pneumatosis intestinalis}

Während der Screening-Koloskopie eines 63-jährigen Mannes fielen im Bereich der linken Flexur mehrere submuköse polypoide Schleimhautvorwölbungen zwischen 3 und $8 \mathrm{~mm}$ Durchmesser auf. Nachdem die Läsionen mit dem Gerät eindrückbar waren und einen bläulichen Farbton hatten, wurde eine Pneumatosis intestinalis vermutet (Abb. A). Bei Punk-tion mit einer Nadel ließen sich die Raumforderungen Pneumatosis intestinalis. entleeren, wodurch die Diagnose bestätigt war.

In der Computertomografie erkannte man zahlreiche luftgefüllte Säckchen der Dickdarmwand im Bereich der linken Fle-
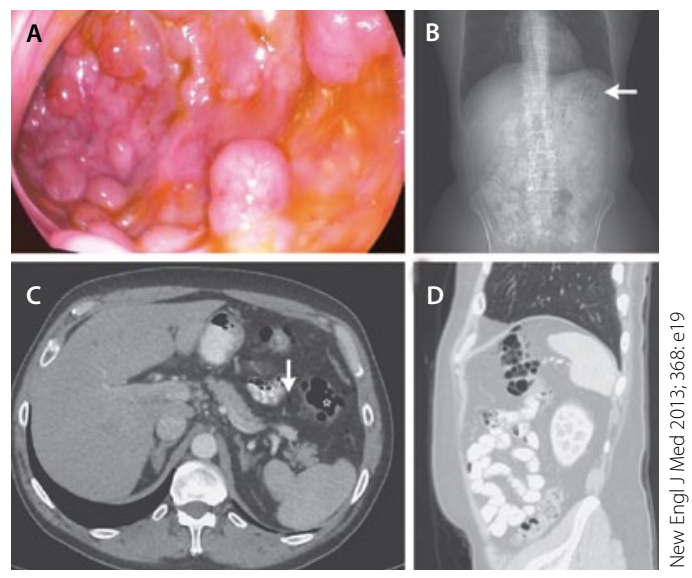

Kommentar

In bestimmten Fällen stellt die Pneumatosis intestinalis einen Surrogatmarker für eine intestinale Ischämie oder eine drohende Perforation dar. Meistens handelt es sich aber um eine gutartige Veränderung, die gar nicht als Erkrankung anzusehen ist, sondern als ein harmloser Befund, dessen Bedeutung jeweils im klinischen Kontext zu interpretieren ist. Bei dem Patienten waren keine therapeutischen Maßnahmen notwendig, zumal er auch völlig asymptomatisch war.

H. S. FÜESSL =

\title{
Was hat der präfrontale Kortex mit Suizid zu tun?
}

\section{Unterscheiden sich depressive Hochrisikopatienten für Suizidalität neurobiologisch von depressiven Patienten ohne erhöhtes suizidales Risiko bzw. von gesunden Kontroll- personen?}

— Die Depression gilt als diejenige psychische Erkrankung, die aufgrund von Hoffnungslosigkeit näher an suizidales Verhalten heranführt. Das Lebenszeitsuizidrisiko für schwer und sehr schwer depressiv kranke Menschen liegt immer noch bei 14-15. Aktuell gibt es einige Hinweise, dass unabhängig von einer Depression eine Prädisposition für suizidales Verhalten selbst existieren mag.

Neuere neurobiologische Studien unterstützen die Beobachtung, dass depressive Patienten mit suizidalem Verhalten möglicherweise ein Muster an neurobiochemischen und neuroanatomischen Veränderungen aufweisen, die sich von depressiven Patienten ohne suizidales Verhalten unterscheiden.

Vor dem Hintergrund der Hypothese, dass bei einer Depression oder auch bei anderen psychischen Erkrankungen zusätzlich neurobiologische Veränderung vorliegen können, die die Prädisposition für suizidales Verhalten noch weiter erhöhen können, wurde nach Differenzen in der kortikalen Dicke über den gesamten Kortex hinweg gesucht. Zentrales Ergebnis war, dass Patienten mit einem Hochrisiko für Suizid einen signifikant dünneren Kortex im linken dorsolateralen, im ventrolateral-präfrontalen Kortex und im anterioren Cingulum zeigten als Patienten ohne Hochrisiko für Suizidalität.

Nach Ansicht der Autoren weisen diese Ergebnisse auf eine starke Evidenz für strukturelle Alterationen des Gehirns bei depressiven Hochrisikopatienten für Suizid im fronto-cingulär-striatalen Netzwerk hin, welches bekanntlich in Belohnungssystem und Verhaltensund Emotionskontrolle eingebunden ist.

\footnotetext{
- G. Wagner et al.

Prefrontal cortical thickness in depressed patients with high-risk for suicidal behaviour. J Psychiatric Res 2012; 46: 1449-1455
}

\section{Kommentar}

Ist Suizidalität eine eigenständige Verhaltens- und Denkweise, unabhängig von depressiven oder anderen psychischen Erkrankungen? So führt zwar Hoffnungslosigkeit grundsätzlich nahe an Suizidalität heran, gerade die Hoffnungslosigkeit in der Depression, insbesondere wenn sie von wahnhaften depressiven Erlebnisweisen gespeist wird. Andererseits kann suizidales Verhalten genauso bei Aggression, Impulskontrollstörung oder bei akut bedrohlich erlebten psychotischen Wahrnehmungen auftreten und umgesetzt werden.

Dass Neurobiologie nur ein Teil der Diagnostik von Suizidalität und Suizidprävention sein kann, braucht an dieser Stelle nicht unterstrichen werden. Dass neurobiologische Aspekte bei Suizidalität eine signifikante Bedeutung haben können, ist aber auch für den Kliniker wichtig. Inwieweit aber hieraus für den Kliniker/Therapeuten im Alltag bereits Konsequenzen zu ziehen sind, muss derzeit noch offen bleiben.

M. WOLFERSDORF a 